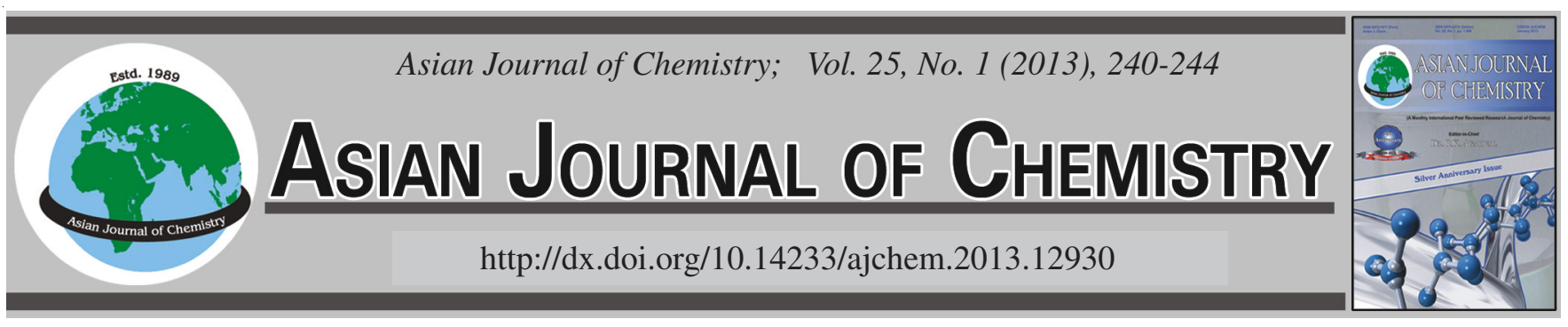

\title{
Facile Biodiesel Synthesis from Esterification of Free Fatty Acids Catalyzed by $\mathrm{SO}_{3} \mathrm{H}$-Functionalized Ionic Liquid
}

\author{
Shuai Zhou ${ }^{1}$, Lu Liu ${ }^{1}$, Bo Wang ${ }^{1, *}$, Feng Xu ${ }^{1}$ and Runcang Sun ${ }^{1,2}$
}

${ }^{1}$ Institute of Biomass Chemistry and Technology, College of Materials Science and Technology, Beijing Forestry University, 100083 Beijing, P.R. China ${ }^{2}$ Key State Laboratory of Pulp and Paper Engineering, South China University of Technology, Guangzhou 510640, P.R. China

*Corresponding author: Fax: +86 10 62336592; Tel: +86 10 62336592; E-mail: mzlwb@ @jfu.edu.cn

The esterifications of free fatty acids with methanol were efficiently accomplished over a $\mathrm{SO}_{3} \mathrm{H}$-functionalized ionic liquid, 1-(4-sulphonic acid)butyl-3-methylimidazolium hydrogen sulphate ([BSMim] $\left.\mathrm{HSO}_{4}\right)$, which are commonly considered as both one of the typical synthetic routes and the representative pretreatment process for biodiesel production. Particularly, the maximum yield of methyl oleate was obtained in $99.9 \%$. Methyl oleate could be isolated through simple decantation from the biphasic system, which is attributed to the immiscibility of $[\mathrm{BSMim}] \mathrm{HSO}_{4}$ with hydrophobic ester and the good stability and operability of ionic liquid were demonstrated by the six times reuses without dramatic decrease in methyl oleate yield.

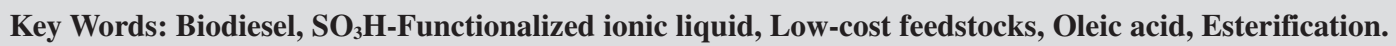

\section{INTRODUCTION}

Biodiesel, a clean-burning diesel fuel with much lower toxic air emissions and biodegradable features ${ }^{1-3}$, usually produced from non-toxic and biologically renewable resources, has been paid much attention all over the world and considered as the best candidate for the substitution of petroleum products in the future ${ }^{4}$. Along with the rapid reduction of arable land and exceeding shortage of resources in the world, effective recycling and reusing low-cost feedstocks for biodiesel production, such as non-edible and waste frying oils, have gradually become one of the significant approaches to reducing the cost of biodiesel production, which is the most vital theme of biodiesel science ${ }^{5}$. However, the high content of free fatty acids in the non-edible oils usually leads to serious processing problems, which require multiple chemical steps or alternate approaches to produce biodiesel and consequently increase the production cost, even reduce the biodiesel yield below the standards ${ }^{6}$. Therefore, an esterification pretreatment of the free fatty acids to alkyl esters in the presence of an acid catalyst could efficiently eliminate the problems. In this regard, the traditionally used acid catalysts such as sulphuric acid, organic acids and solid acids etc., are usually associated with one of the following disadvantages such as high temperature, long reaction time, high cost and moisture sensitivity of the catalyst, tedious isolation procedures, equipment corrosion and environmental problems ${ }^{7,8}$. In order to abate these deficiencies, several alternative technologies such as using heterogeneous catalysts ${ }^{9}$, enzyme $^{10}$ and membrane ${ }^{11}$ have been investigated. However, the relatively high cost or complexity and instability of catalytic system unquestionably restrains their wide applications and large-scale production.

In pace with the intensive study for task-specific ionic liquids, a great range of acid-functionalized ionic liquids tendentiously emerged as special acidic catalysts to perfect and improve the organic synthesis ${ }^{12}$, especially including biodiesel synthesis process ${ }^{13}$. Neto et al. ${ }^{14}$ has developed a biphasic catalytic system that tin complexes is immobilized in 1- $n$-butyl-3-methylimidazolium tetrachloro-indate ([BMim] $\mathrm{InCl}_{4}$ ) ionic liquid. However, the insuperable defects in this system such as the metal componet leaching and non-reusability of catalyst usually impair this methodology. Although the biodiesel synthesis from soybean oil has been proceeded using $\left[\mathrm{Et}_{3} \mathrm{NH}\right] \mathrm{Cl}-\mathrm{AlCl}_{3}$ as catalyst reported by Yang et al. ${ }^{15}$. It is well known that the chloroaluminate ionic liquids could not be applied to the large-scale production just due to its sensitivity to air and water. Nevertheless, the research and application of various $\mathrm{SO}_{3} \mathrm{H}$-functionalized ionic liquids with strong Brønsted acidicity have received much attention due to their potential applications in replacing conventionally catalytic systems ${ }^{16,17}$. In the research of biodiesel synthesis, $\mathrm{SO}_{3} \mathrm{H}$-functionalized ionic liquids have revealed the peculiar characteristics that they could serve as both acid catalyst and reaction media, leading to simplify the product isolation and achieve the catalysts' 
recovering and recycling. In a particular case, the biodiesel preparation from waste oils catalyzed by pyridine-based sulphonic acid-functionalized ionic liquids has been reported, however the relatively lower catalytic activities have to bring on the harsh reaction conditions ${ }^{18,19}$.

During the course of the preparation of high value materials from biomass, we found that the imidazolium-based $\mathrm{SO}_{3} \mathrm{H}$-functionalized ionic liquids have been proved to be the excellent acidic catalytic media and applied to many kinds of organic reactions due to their inherent strong acidity and thermal stability ${ }^{20}$. This prompted us to investigate the esterification of free fatty acids as the model pretreatment process of biodiesel synthesis catalyzed by 1-(4-sulphonic acid)butyl-3methylimidazolium hydrogen sulphate ([BSMim] $\left.\mathrm{HSO}_{4}\right)$ ionic liquid for the purpose of exploiting the low-cost feedstocks and oleic acid and methanol were designated as the model substrates for the esterification reaction as shown in Scheme-I.

\section{EXPERIMENTAL}

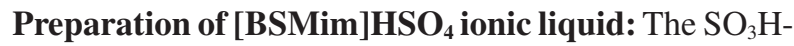
functionalized ionic liquid [BSMim] $\mathrm{HSO}_{4}$ was synthesized according to the literature method ${ }^{21}$. The detailed preparation procedure was as follows: To $150 \mathrm{~mL}$ toluene in $500 \mathrm{~mL}$ threenecked round-bottom flask equipped with mechanical stirrer, 1,4-butanesulfone $(51.1 \mathrm{~mL}, 0.5 \mathrm{~mol})$ was added followed by an equal-mole $\mathrm{N}$-methylimidazole $(39.6 \mathrm{~mL}, 0.5 \mathrm{~mol})$ transferring at room temperature and the mixture was kept stirring for overnight to form white precipitate. Then the white solid was filtered out, washed with diethyl ether thrice $(30 \mathrm{~mL} \times$ $3 \mathrm{~mL}$ ) to remove non-ionic residues and dried at $80^{\circ} \mathrm{C}$ under vaccum for overnight to get the zwitterion, 4-(1-methylimidazolium-3-yl)butane-1-sulfonate (yield 97.1\%). ${ }^{1} \mathrm{H}$ NMR (400 MHz, $\left.\mathrm{D}_{2} \mathrm{O}\right): \delta 1.670(\mathrm{~m}, 2 \mathrm{H}), 1.977(\mathrm{~m}, 2 \mathrm{H}), 2.878(\mathrm{~m}$, 2H), 3.851 (s, 3H, J = 3.0 Hz), $4.203(\mathrm{~m}, 2 \mathrm{H}), 7.414(\mathrm{~m}, 1 \mathrm{H})$, $7.475(\mathrm{~m}, 1 \mathrm{H}), 8.710(\mathrm{~s}, 1 \mathrm{H})$.

Nextly, the white zwitterion was charged into $100 \mathrm{~mL}$ $\mathrm{H}_{2} \mathrm{O}$ in $500 \mathrm{~mL}$ round-bottom flask and a stoichiometric amount of concentrated sulphuric acid was added dropwise at room temperature under vigorously magnetic stirring. Then, the reaction mixture was gradually heated up to $90{ }^{\circ} \mathrm{C}$ and kept stirring for $8 \mathrm{~h}$ followed by removing water under vacuum at $90{ }^{\circ} \mathrm{C}$, giving $\left[\mathrm{BSMim} \mathrm{HSO}_{4}\right.$ ionic liquid (yield 98.0\%). ${ }^{1} \mathrm{H}$ NMR (400 MHz, DMSO): $\delta 1.586(\mathrm{~m}, J=7.32 \mathrm{~Hz}, 2 \mathrm{H})$, $1.905(\mathrm{~m}, J=7.44 \mathrm{~Hz}, 2 \mathrm{H}), 2.624(\mathrm{t}, J=7.56 \mathrm{~Hz}, 2 \mathrm{H}), 3.873$ (s, 3H), $4.204(\mathrm{t}, J=7.04 \mathrm{~Hz}, 2 \mathrm{H}), 7.724(\mathrm{~m}, J=1.64 \mathrm{~Hz}$, 1H), 7.786 (m, $J=1.64 \mathrm{~Hz}, 1 \mathrm{H}), 9.177$ (s, 1H), 9.294 (bs, 2H).

Esterification of oleic acid and methanol catalyzed by [BSMim] $\mathrm{HSO}_{4}$ : The esterification of oleic acid with methanol catalyzed by $\left[\mathrm{BSMim} \mathrm{HSO}_{4}\right.$ was investigated as the model pretreating reaction for the biodiesel synthesis derived from the high-acid value waste oils. A special procedure: [BSMim] $\mathrm{BSO}_{4}(2.0 \mathrm{~g}, 6.3 \mathrm{mmol})$ was charged into a $50 \mathrm{~mL}$ round-bottom flask followed by the addition of oleic acid (10 mL, $31.6 \mathrm{mmol})$ and $\mathrm{MeOH}(2.6 \mathrm{~mL}, 63.2 \mathrm{mmol})$ under magnetic stirring. Then the mixture was heated to $80{ }^{\circ} \mathrm{C}$ and kept stirring for desired duration time. The biphasic system formed when the stirrer stopped. The upper phase containing methyl oleate and unreacted oleic acid was isolated by simple decantation. And the ionic liquid phase retaining methanol is always the lower phase, which can be recovered after simple treatment under vacuum and used directly for the next run. The identification of samples was detected from ${ }^{1} \mathrm{H}$ NMR spectra, which were recorded on a MERCURY-PLUS 400 NMR spectrometer.

\section{RESULTS AND DISCUSSION}

Activity comparison of different acidic catalysts for esterification of oleic acid: Initially, an activity comparison study was carried out with several catalysts and ionic liquids which are similar with chemical structure, in the esterification of oleic acid and the results are summarized in Table-1. It can be seen that, $\mathrm{H}_{2} \mathrm{SO}_{4}$ as the common inorganic acid catalyst gives rise to a good ester yield $(96.3 \%)$, which is much better than that from the organic acid catalysts such as $p$-toluenesulphonic acid ( $p$-TSA, $38.2 \%$ ) and sulfamic acid $\left(\mathrm{NH}_{2} \mathrm{SO}_{3} \mathrm{H}\right.$, $68.1 \%$ ), respectively under the same reaction conditions. However, the toxicity, corrosivity and contamination of these catalysts forced people to use the more benign alternatives. Herein, the sulphonic acid functionalized ionic liquids were chosen as the friendly catalyst for the esterification of oleic acid and methanol. And $[\mathrm{BSMim}] \mathrm{HSO}_{4}$ ionic liquid shows better reactivity than $p$-TSA and $\mathrm{NH}_{2} \mathrm{SO}_{3} \mathrm{H}$. Methyl oleate yield could come up to $92.0 \%$, which is undoubtedly better than that over $\mathrm{H}_{2} \mathrm{SO}_{4}$ from the viewpoint of environmental protection, although there is a little decrease in ester yield comparatively. While the ionic liquids with the same cations [BSMim] $\mathrm{H}_{2} \mathrm{PO}_{4}$ and [BSMim]OAc, just only provide the ester yields in 33.5 and $17.2 \%$, respectively, even if the reaction time is extended twice. At the same time, the negligible ester yield $(3.4 \%)$ was achieved when reaction was carried out only using [BMim] $\mathrm{Cl}$ ionic liquid as catalyst. This demonstrated that the butyl sulphonic acid group attached to the cation has relatively little influence on the esterification of oleic acid. On the contrary, the anions of ionic liquids decisively predominate their own catalytic performance depending on the corresponding acid strength of anions ${ }^{22}$.

Additionally, it is well known that current biodiesel production in industry through alkali-catalyzed transesterification using $\mathrm{KOH}, \mathrm{NaOH}$ or $\mathrm{K}_{2} \mathrm{O}$ as catalysts, usually suffered from the saponification, which entirely contributes to the decrease in the biodiesel yield and catalyst efficiency ${ }^{23}$. Based on this,
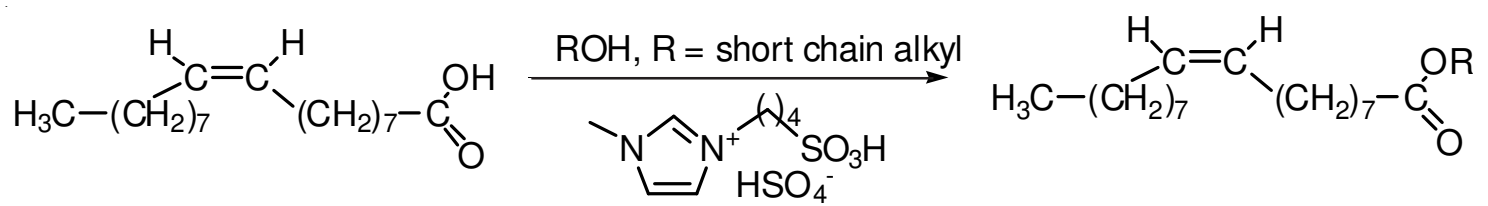

Scheme-I: Model biodiesel synthesis over esterification of oleic acid and methanol catalyzed by $\left[\mathrm{BSMim} \mathrm{HSO}_{4}\right.$ 


\begin{tabular}{|c|c|c|c|}
\hline \multicolumn{4}{|c|}{$\begin{array}{c}\text { TABLE-1 } \\
\text { COMPARISON OF ESTERIFICATION OF OLEIC ACID AND } \\
\text { MeOH IN THE PRESENCE OF DIFFERENT CATALYSTS }\end{array}$} \\
\hline Catalyst & Cat. $(\mathrm{mol} \%)^{\mathrm{b}}$ & Time (h) & Yield of ester $(\%)^{c}$ \\
\hline $\mathrm{H}_{2} \mathrm{SO}_{4}$ & 40 & 6 & 96.3 \\
\hline $\mathrm{NH}_{2} \mathrm{SO}_{3} \mathrm{H}$ & 40 & 6 & 68.1 \\
\hline$p$-TSA & 40 & 6 & 38.2 \\
\hline [BMim] Cl & 40 & 24 & 3.4 \\
\hline$[\mathrm{BMim}] \mathrm{ClH}_{2} \mathrm{SO}_{4}$ & $40 / 40$ & 6 & 97.5 \\
\hline$[\mathrm{BMim}] \mathrm{HSO}_{4}$ & 40 & 6 & 84.8 \\
\hline$[\mathrm{BSMim}] \mathrm{HSO}_{4}$ & 40 & 6 & 92.0 \\
\hline$[\mathrm{BSMim}] \mathrm{H}_{2} \mathrm{PO}_{4}$ & 40 & 16 & 33.5 \\
\hline [BSMim]Ac & 40 & 16 & 17.2 \\
\hline [BMim]OH & 100 & 16 & 15.7 \\
\hline
\end{tabular}

we have expected the basic ionic liquid [BMim]OH (1-butyl3 -methylimidazolium hydroxide $)^{24}$ would eradicate the saponification problem. However, it seems that the elementary exploration is not successful (entry 7), the activity of [BMim] $\mathrm{OH}$ has been destroyed on account of the interaction between ionic liquid and fatty acid.

Reaction conditions optimization: We set out to examining the effect of reaction temperature, time, catalyst amount, molar ratio of reactants on the esterification of oleic acid and methanol in the presence of $\left[\mathrm{BSMim} \mathrm{HSO}_{4}\right.$. As shown in Fig. 1, 62.5\% of ester yield was achieved after $6 \mathrm{~h}$ at room temperature, indicating the inherent efficiency of the acidic ionic liquid. For a $20^{\circ} \mathrm{C}$ rise in temperature, the yield of methyl oleate could increase about $10 \%$ during the same reaction time. Consequently, when the reaction was performed under reflux $\left(80^{\circ} \mathrm{C}\right.$ in oil bath), the yield of methyl oleate could reach the maximum $(92 \%)$.

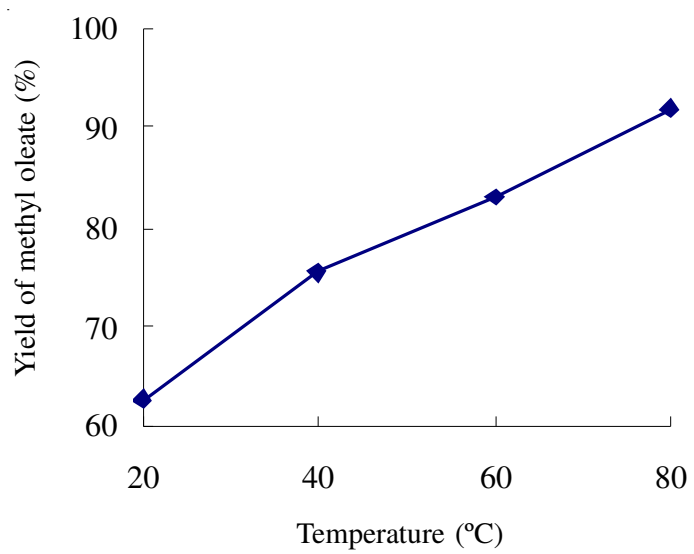

Fig. 1. Effect of reaction temperature on the esterification of oleic acid with $\mathrm{MeOH}$ catalyzed by $[\mathrm{BSMim}] \mathrm{HSO}_{4}$ (oleic acid: $\mathrm{MeOH}=1: 2$; $40 \mathrm{~mol} \%$ of ionic liquid; $6 \mathrm{~h}$ )

As the data shown in Fig. 2, the reaction proceeded fast at the beginning and $85.2 \%$ of ester yield was obtained after $4 \mathrm{~h}$ at $80{ }^{\circ} \mathrm{C}$. When reaction time was prolonged to $8 \mathrm{~h}, 94.6 \%$ of methyl oleate was achieved. This result was comparable to the yield data $(95 \%)$ using $[\mathrm{NMP}] \mathrm{CH}_{3} \mathrm{SO}_{3}$ ionic liquid as acidic catalyst $^{25}$. Subsequently, the yield of methyl oleate increased slowly and finally the reaction nearly reached to completion $(99.9 \%)$ at $18 \mathrm{~h}$. Taking the energy consumption into account,

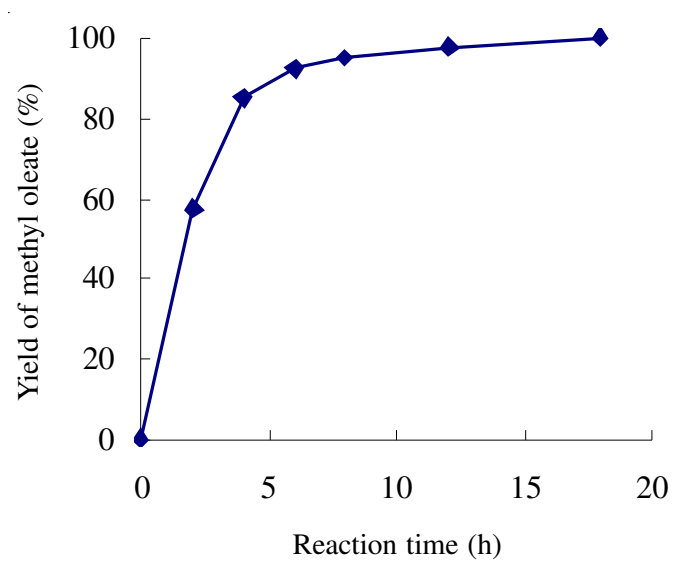

Fig. 2. Effect of reaction time on the esterification of oleic acid with $\mathrm{MeOH}$ catalyzed by $\left[\mathrm{BSMim}^{-} \mathrm{HSO}_{4}\right.$ (oleic acid: $\mathrm{MeOH}=1: 2 ; 40 \mathrm{~mol} \%$ of $[\mathrm{BSMim}] \mathrm{HSO}_{4} ; 80{ }^{\circ} \mathrm{C}$ )

$12 \mathrm{~h}$ could be optimal for the esterification $(97.5 \%)$ of oleic acid with methanol catalyzed by $[\mathrm{BSMim}] \mathrm{HSO}_{4}$ ionic liquid. Fig. 3 revealed that as the proportion of ionic liquid increasing, a significant rise in the initial rate was observed when the ionic liquid amount was less than $15 \mathrm{~mol} \%$ (based on substance). However, further improving the proportion of ionic liquid to $40 \mathrm{~mol} \%$ based on oleic acid, the increased rate of product yield started to decrease slightly. For a rise in content of ionic liquid from $15-40 \mathrm{~mol} \%$, the yield of methyl oleate just only increased about $1.7 \%$ under the same reaction conditions. Moreover, it looks like that too much ionic liquid has a detrimental effect on the ester yield (100 mol \% of [BSMim] $\mathrm{HSO}_{4}$ based on oleic acid, $93.3 \%$ of ester yield), which is probably due to the relatively poor dispersion of ionic liquid in the reaction mixture. As far as the reusability of ionic liquid is concerned, $20 \mathrm{~mol} \%$ proportion of ionic liquid was chosen as the best option.

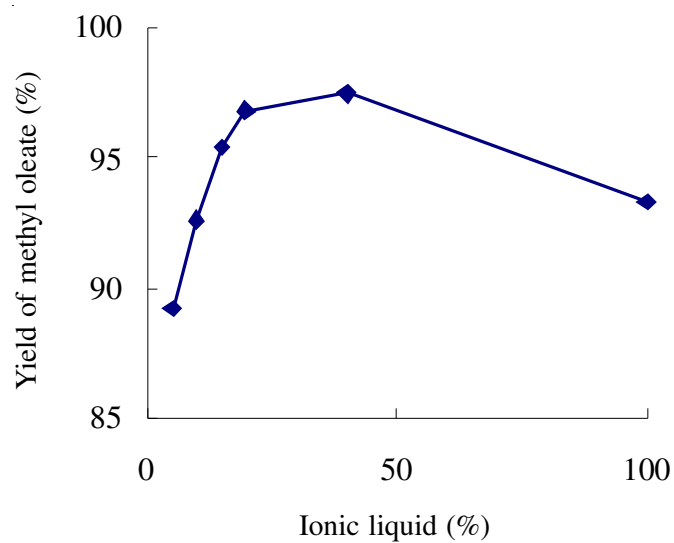

Fig. 3. Effect of ionic liquid amount on the esterification of oleic acid with $\mathrm{MeOH}$ catalyzed by $[\mathrm{BSMim}] \mathrm{HSO}_{4}\left(80^{\circ} \mathrm{C} ; 12 \mathrm{~h}\right.$; oleic acid: $\mathrm{MeOH}$ $=1: 2)$

Undoubtedly, an excess of alcohol is necessary for the esterification of oleic acid. However, a cost-efficient molar ratio of $\mathrm{MeOH}$ to oleic acid should be favorable for the practical application of the catalytic system. Fig. 4 showed that the yield of methyl oleate increased slightly from 97.5-99.5\% with the molar ratio of methanol to oleic acid increasing from 2:1 to $10: 1$. Thus, it seems that too much excess of methanol 


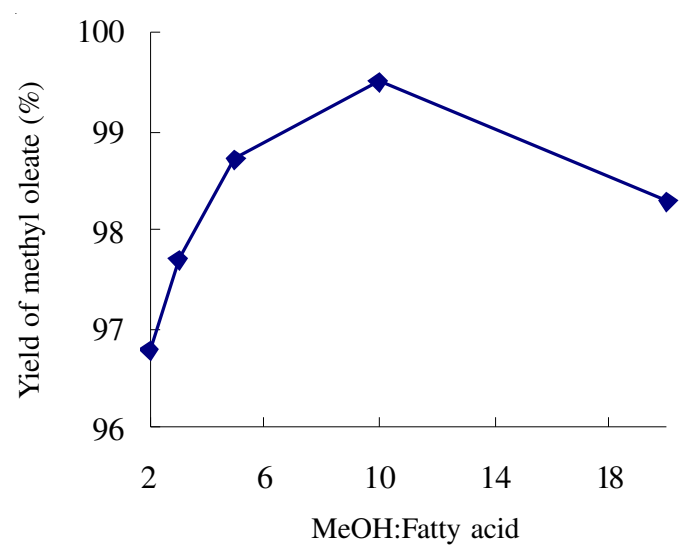

Fig. 4. Effect of molar ratio of $\mathrm{MeOH}$ to oleic acid on the eaterification of oleic acid with $\mathrm{MeOH}$ catalyzed by $\left[\mathrm{BSMim}^{-} \mathrm{HSO}_{4}(20 \mathrm{~mol} \%\right.$ of $\left[\right.$ BSMim] $\mathrm{HSO}_{4} ; 80^{\circ} \mathrm{C} ; 12 \mathrm{~h}$ )

is not always good for the production of biodiesel, probably due to the dilution effect of [BSMim] $\mathrm{HSO}_{4}$ catalyst by methanol. The similar phenomenon has been displayed by Zhang et al. ${ }^{25}$. Therefore, the molar ratio of 2:1 was believed to be optimal in the $[\mathrm{BSMim}] \mathrm{HSO}_{4}$ catalytic system with regard to the production cost. It is worth mentioning that increasing the amount of methanol did not influence the biphasic system formation after reaction and the product ester still could be separated easily by decantation.

Reusability of [BSMim] $\mathrm{HSO}_{4}$ in esterfication of oleic acid: In this study, $\left[\mathrm{BSMim} \mathrm{HSO}_{4}\right.$ ionic liquid was separated from the organic phase after first run and treated under vacuum before it was directly used in the next run. From the results given in Fig. 5, there is no dramatic decrease in ester yield after [BSMim] $\mathrm{HSO}_{4}$ was reused six times in the esterification of oleic acid, which gives evidence of the thermal stability and feasibility of [BSMim] $\mathrm{HSO}_{4}$ ionic liquid for industrial production of biodiesel.

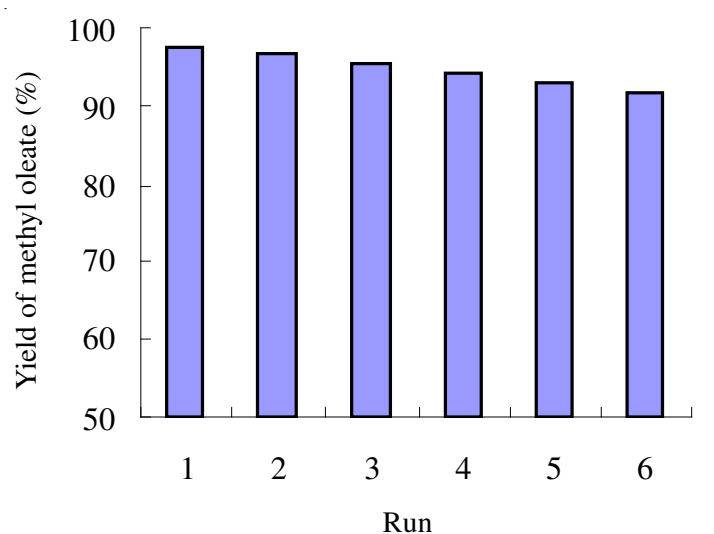

Fig. 5. Reuse of $\left[\mathrm{BSMim}^{-} \mathrm{HSO}_{4}\right.$ in the esterification of oleic acid with $\mathrm{MeOH}\left(40 \mathrm{~mol} \%\left[\mathrm{BSMim} \mathrm{HSO}_{4}\right.\right.$; oleic acid: $\mathrm{MeOH}=1: 2 ; 80{ }^{\circ} \mathrm{C}$; $12 \mathrm{~h})$

Esterifications of other fatty acids catalyzed by [BSMim] HSO $_{4}$ : It is well known that there are many kinds of fatty acids existing in oils and fats. Moreover, high content of free fatty acids was detected in non-edible oils and waste frying oils ${ }^{26}$, which usually leads to significant processing problems, including multiple chemical steps or alternation of approaches to producing biodiesel, increase in the production cost and depression of biodiesel quality below the standards ${ }^{6}$. Encouraged by the promising results in hand, we have attempted to perform the esterification of several other fatty acids e.g., (lauric acid $98.3 \%$, myristic acid $97.4 \%$, palmitic acid $95.7 \%$ and stearic acid $95.6 \%$ ) with methanol under the above-mentioned optimized conditions. Evidently, these data revealed that the alkyl chain length of fatty acid has negligible little effect on the esterification of long-chain fatty acids. Accordingly, it can be said that all of the free fatty acids involved in the waste frying oils could be converted into esters in the presence of [BSMim] $\mathrm{HSO}_{4}$, no matter what kind of fatty acids they are, which is very valuable for the effective recycling and reusing of low-cost feedstocks.

\section{Conclusion}

In summary, esterification of fatty acids and methanol has been performed smoothly using $[\mathrm{BSMim}] \mathrm{HSO}_{4}$ ionic liquid (20 mol \%) as catalyst and methyl oleate could be obtained with $96.8 \%$ of yield at $80{ }^{\circ} \mathrm{C}$ after $12 \mathrm{~h}$, which is commonly deemed as both one of the typical synthetic routes and the representative pretreatment process for biodiesel production. Furthermore, the reactivity of sulphonic acid functionalized ionic liquids for the esterification of oleic acid was probably predominated by the anions paired to imidazolium cation. Particularly, the product separation was easily accomplished through the decantation of the biphasic system attributed to the immiscibility of the acidic ionic liquid with organic ester. Moreover, [BSMim] $\mathrm{HSO}_{4}$ could be reused several times just only to need simple vacuum treatment. This method is far superior to others performed using toxic acidic catalysts such as $\mathrm{H}_{2} \mathrm{SO}_{4}$, because [BSMim] $\mathrm{HSO}_{4}$ ionic liquid has combined the advangtages of friendly benign acid catalyst with an excellent product separation methodology, as well as the cost efficiency and green aspect, which are in line with the demand of sustainable chemistry.

\section{ACKNOWLEDGEMENTS}

Financial supports from the Fundamental Research Funds for the Central Universities (YX2011-36, TD2011-1, TD2010-1), the Forestry University Young Scientist Fund (BLX2009003), National Natural Science Foundation of China (31170556), Research Fund for the Doctoral Program of Higher Education of China (20100014120007), China Postdoctoral Science Foundation (20110490303), Major State Basic Research Development Program of China (973 Program, No. 2010CB 732204) and China Ministry of Education (No. 111 project) are gratefully acknowledged.

\section{REFERENCES}

1. H.J. Berchmans and S. Hirata, Bioresour. Technol., 99, 1716 (2008).

2. S.P. Singh and D. Singh, Renew. Sustan. Energ. Rev., 14, 200 (2009).

3. P. Schinas, G. Karavalakis, C. Davaris, G. Anastopoulos, D. Karonis, F. Zannikos and S. Stournas, Biomass Bioenerg., 33, 44 (2009).

4. J.M. Marchetti, V.U. Mguel and A.F. Errazu, Renew. Sustan. Energ. Rev., 11, 1300 (2007).

5. J.M. Dias, M.C.M. Alvim-Ferraz and M.F. Almeida, Bioresour. Technol., 100, 6355 (2009).

6. P.D. Patil and S. Deng, Fuel, 88, 1302 (2009).

7. Y. Rezgui and M. Guemini, Ind. Eng. Chem. Res., 12, 4056 (2008). 
8. G. Antolín, F.V. Tinaut, Y. Briceño, V. Castaño, C. Pérez and A.I. Ramírez, Bioresour. Technol., 83, 111 (2002).

9. M.D. Serio, M. Cozzolino, R. Tesser, P. Patrono, F. Pinzari, B. Bonelli and E. Santacesaria, Appl. Catal. A: Gen., 320, 1 (2007).

10. M.M. Teresa, A.A. Martins and N.S. Caetano, Renew. Sust. Energ. Rev. 14, 217 (2010)

11. M.A. Dubé, A.Y. Tremblay and J. Liu, Bioresour. Technol., 98, 639 (2007).

12. Z.S. Qureshi, K.M. Deshmukh, M.D. Bhor and B.M. Bhanage, Catal. Commun., 6, 833 (2009)

13. H. Zhao, Z.Y. Song, O. Olubajo and J.V. Cowins, Appl. Biochem. Biotechnol., 162, 13 (2010).

14. B.A. DaSilveira Neto, M.B. Alves, A.A.M. Lapis, F.M. Nachtigall, M.N Eberlin, J. Dupont and P.A.Z. Suarez, J. Catal., 249, 154 (2007).

15. X.Z. Liang, G.Z. Gong, H.H. Wu and J.G. Yang, Fuel, 88, 613 (2009).

16. D. Fang, J. Cheng, Z. Fei, K. Gong and Z. Liu, Catal. Commun., 9, 1924 (2008)
17. J. Akbari and A. Heydari, Tetrahedron Lett., 29, 4236 (2009).

18. M.H. Han, W.L. Yi, Q. Wu, Y. Liu, Y.C. Hong and D.Z. Wang, Bioresour. Technol., 100, 2308 (2009).

19. X.Z. Liang and J.G. Yang, Green Chem., 12, 201 (2010).

20. J.H. Shen, H. Wang, H.C. Liu, Y. Sun and Z.M. Liu, J. Mol. Catal. A: Chem., 280, 24 (2008).

21. A.C. Cole, J.L. Jensen, L. Ntai, K.L.T. Tran, K.J. Weaver, D.C. Forbes and J.H. Davis, J. Am. Chem. Soc., 124, 5962 (2002).

22. H. Xing, T. Wang, Z. Zhou and Y. Dai, Ind. Eng. Chem. Res., 11, 4147 (2005).

23. D.Y.C. Leung, X. Wu and M.K.H. Leung, Appl. Energ., 87, 1083 (2010).

24. B.C. Ranu and S. Banerjee, Org. Lett., 14, 3049 (2005).

25. L. Zhang, M. Xian, Y.C. He, L.Z. Li, J.M. Yang, S.T. Yu and X. Xu, Bioresour. Technol., 100, 4368 (2009).

26. M.P. Dorado, E. Ballesteros, J.M. Arnal, J. Gòmez and F.J.L. Gimènez, Energ. Fuel, 17, 1560 (2003). 